\title{
Restricting and Embedding Imperatives
}

\author{
Nate Charlow \\ Department of Philosophy, University of Michigan \\ ncharlo@umich.edu
}

\begin{abstract}
We use imperatives to refute a naïve analysis of update potentials (force-operators attaching to sentences), arguing for a dynamic analysis of imperative force as restrictable, directed, and embeddable. We propose a dynamic, non-modal analysis of conditional imperatives, as a counterpoint to static, modal analyses (e.g., Schwager [16]). Our analysis retains Kratzer's [8] analysis of if-clauses as restrictors of some operator (with Schwager), but avoids typing it as a generalized quantifier over worlds (against her), instead as a dynamic force operator (cf. Portner 13. 14]; Potts 15]). Arguments for a restrictor treatment (but against a quantificational treatment) are mustered, and we propose a novel analysis of update on conditional imperatives (and an independently motivated revision of the standard ordering-semantics for root modals that makes use of it). Finally, we argue that imperative force is embeddable under an operation much like dynamic conjunction.
\end{abstract}

\section{Plan}

Sentences of the imperative clause-type (hereafter 'imperatives') are conventionally associated with a distinctive kind of force (what I will call 'imperative force') that is both performative and directive (see esp. Portner [13]). It is performative in the sense that the conventional discourse function of imperatives is not to describe facts about the world, but rather to introduce new facts (about obligations or commitments) into a discourse. It is directive in the sense that imperatives function primarily to shape the intentions (indirectly, by directly shaping things that, in turn, directly shape the intentions) of their addressees.

There is widespread agreement that a semantico-pragmatic analysis of imperatives should have something to say about this dimension (call it the 'force dimension') of the conventional meaning of imperatives 1 What, exactly, needs to be said, beyond the fact that imperatives conventionally receive performative and directive interpretations, is often unclear. In this paper, I articulate

\footnotetext{
${ }^{1}$ Some (e.g., Portner [14]: 366) have taken the stronger position that the unavailability of non-performative interpretations of imperatives means that the force dimension exhausts the dimensions of imperative meaning. This latter position is too strong. As I argue in [2, there are dimensions of imperative meaning (e.g., facts about their inferential and logical properties) that are paradigmatically static and do not emerge straightforwardly from an account of the force dimension.
}

M. Aloni et al. (Eds.): Amsterdam Colloquium 2009, LNAI 6042, pp. $223-233,2010$.

(C) Springer-Verlag Berlin Heidelberg 2010 
substantive conditions of adequacy on an account of the force dimension of imperative meaning. My principal focus is on the performative effects of conditional imperatives (CIs; see 1) and unconditional imperatives (UIs; see 2).

(1) If the temperature drops, shut the window! $\approx($ if $\phi)(! \psi)$

(2) Shut the window! $\approx ! \phi$

Schwager's [16] account, which treats imperatives as a species of modal clause (hence, imperative operators as Kratzer-ian restrictable modal operators), is designed to handle CIs, but ultimately handles neither. Portner's 14 account makes implicit use of directed speech-act (force) operators (à la Potts [15]), so that the force of an imperative is to add the content of the imperative (what's commanded, i.e., the complement of the force operator) to the addressee's To-Do List. It does well with UIs, but falters with CIs.

We strictly improve on these proposals by reconceptualizing force operators. The ordinary treatment (classic references are Stenius [17]; Lewis [11]) views speech-acts on the model of propositional attitudes: as an agent may believe $\phi$, she may assert $\phi$, command $\phi$, question whether $\phi$, etc 2 Handling CIs requires a new approach: speech-acts are less like propositional attitudes, more like literal actions whose force (contextual effect) can be modulated, via linguistic and extra-linguistic mechanisms, and whose functional potential can be formally modeled in a familiar logic of programs. Conditional imperatives, we'll see, illustrate a syntactic mechanism of force-modulation, which we model as force-restriction (in a sense to be precisified). (Making use of this analysis requires modifying the standard Kratzer [8] semantics for modals. There are, we'll see, independent reasons for doing this.)

Our stance here resembles that of Krifka [9, 10, which emphasizes natural language devices (generally corresponding to regular operations on programs) for building complex speech-acts out of component speech-acts. The question naturally arises: which such operations are expressible in natural language? We end with some tentative remarks on this question.

\section{Menu}

The conventional discourse function of an imperative clause is, I will suppose, to introduce some sort of obligation or commitment on its addressee, via modification of parameters of the context to which the interpretation of obligation- or commitment-describing modalities is sensitive (cf. Han [5],

\footnotetext{
${ }^{2}$ See Krifka 10 . The traditional idea might be motivated by the idea that there is some sort of map from speech-acts onto propositional attitudes: every speech-act expresses some propositional attitude, and speech-act types are individuated by the sort of attitude they generally function to express - asserting that $\phi$ expresses belief that $\phi$, questioning whether $\phi$ expresses wondering whether $\phi$, etc.
} 
Portner [13, 14] $]_{3}^{3}$ Imperative force is performative because it generally yields a context in which certain obligation-descriptions are true (where previously they were false), directive because its target is the indirect regulation of the behavior of its addressee. Adequate accounts of imperative force will predict that CIs tend to introduce corresponding conditional obligations (COs), UIs tend to introduce corresponding unconditional obligations (uOs). Concretely, (11) and (2) should tend to make it the case that if the temperature drops, you must shut the window, and that you must shut the window, respectively. Should an account fail to predict this in a given context, there should be a plausible explanation (for instance, the prior context enforcing a conflicting obligation).

\subsection{Modal Analyses}

The paradigm modal analysis of imperatives is Schwager's [16]. Schwager assigns a CI (if $\phi)(! \psi)$ the logical form $O(\psi / \phi)$ (read: if $\phi, \psi$ must be realized); UIs are trivially restricted, so that $! \phi:=O(\phi / \top)$. These LFs are interpreted via the standard Kratzer 8 ordering-restrictor semantics. On that semantics, if $c$ is a context, then $f_{c}$ is the modal base (a body of information), $g_{c}$ the ordering source (for Schwager a set of contextually given preferences, generally supplied by the speaker). Both $f_{c}$ and $g_{c}$ map worlds to sets of propositions. The modal LFs are assigned the following truth-conditions 4

Definition 1. $O O(\psi / \phi) \rrbracket^{c, w}=1 \Leftrightarrow \min \left(f_{c}(w) \cup\left\{\llbracket \phi \rrbracket^{c}\right\}, \preceq_{g_{c}(w)}\right) \subseteq \llbracket \psi \rrbracket^{c}$, where:

1. $\min \left(\Phi, \preceq_{\Psi}\right):=\left\{w \in \bigcap \Phi: \forall v \in \bigcap \Phi: v \preceq_{\Psi} w \Rightarrow w \preceq_{\Psi} v\right\}$

2. $w \preceq_{\Psi} v \Leftrightarrow\{P \in \Psi: v \in P\} \subseteq\{P \in \Psi: w \in P\}$

The analysis thus assigns imperatives truth-conditions - the same as their modal LFs. As such, the analysis would appear to offer no account of imperative forceappear, indeed, to predict that imperative force is a subtype of assertoric force.

Schwager tries to avoid the worry by introducing contextual constraints on the felicitous utterance of an imperative. Imperative utterances are infelicitous at $c$ unless the speaker of $c$ :

1. Has exhaustive knowledge, à la Groenendijk and Stokhof [4, about $f_{c}$ and $g_{c}$, so that he 'utters a necessity proposition he cannot be mistaken about.'

2. Affirms the relevant preference for $\phi$ a good 'maxim for acting.'

When these conditions are met, an imperative utterance will generally receive the performative and directive interpretation adverted to above.

There are, however, deep problems. First, if the speaker of $c$ isn't mistaken, then $O(\psi / \phi)$ must already be true at $c$. Performative effect, which paradigmatically consists in updating $c$ so that $O(\psi / \phi)$ goes from false to true, is therefore

\footnotetext{
${ }^{3}$ Some non-imperative clauses (e.g., explicit performatives of the form I hereby command you to...) plausibly have conventionalized imperative force. While this complicates the posited link between clause-type and conventional force, it does not defeat it. Space prevents me from discussing such cases in any detail here, but see Asher and Lascarides 1 for relevant discussion.

4 The Limit Assumption simplifies our discussion (with no worrying commitments).
} 
erased. Second, affirmation that $\phi$ is a good 'maxim for acting' is exactly the type of speech-act we should like to analyze. We would like to model how such affirmation is generally associated with the introduction of new obligations on the addressee. Saying that imperatives receive a performative and directive interpretation when certain presuppositions are met is no replacement for an account of what, precisely, such an interpretation consists in.

\subsection{Dynamic Analyses}

Portner [13, 14] (cf. Han [5]; Potts [15]) analyzes imperative performative effect as addition to an addressee-indexed ordering source, her 'To-Do List' (TDL). Imperative clauses are associated directly with a type of 'sentential force,' rather than indirectly (via analysis as a species of necessity modal with an exclusively performative interpretation). With [.] a dynamic interpretation function, mapping formulas to update potentials, $c$ a context, $T_{c}$ a function from individuals to their TDLs, $a_{c}$ the addressee, the idea is this:

Definition 2. $c[! \phi]=c^{\prime}$ is just like $c$, except $\llbracket \phi \rrbracket^{c}$ is on $T_{c^{\prime}}\left(a_{c}\right)$

This analysis meets the criteria of adequacy on accounts of unconditional imperative force. Making use of the Kratzer semantics for modals, we can see that even where some of the $T_{c}\left(a_{c}\right)$-best worlds compatible with the $c$-relevant information do not satisfy $\phi$, it will tend to be the case that $O(\phi / \top)$ is true at $c^{\prime}$, since it will tend to be the case that all of the $T_{c^{\prime}}\left(a_{c}\right)$-best worlds compatible with the same information do satisfy $\phi$, in virtue of the presence of $\llbracket \phi \rrbracket^{c}$ on $T_{c^{\prime}}\left(a_{c}\right)$. In cases where this does not reliably hold at $c^{\prime}$ (e.g., cases where updating $c$ with ! $\phi$ introduces a logical incompatibility into the TDL), it's not clear that we really do want to predict that new obligations are imposed. Such cases will tend to coincide with cases where the prior context enforces a conflicting obligation.

The analysis does not, however, meet the criteria of adequacy on accounts of conditional imperative force. In a footnote, Portner [13] moots an analysis in terms of conditional update: informally, he suggests, $[($ if $\phi)(! \psi)]$ adds $\llbracket \phi \rrbracket^{c}$ to $a_{c}$ 's TDL, once $\phi$ is true. But this fails to explain the conventional discourse effect of CIs: the imposition of COs. Even when $\phi$ is false at both $c$ and the result of updating $c$ with (if $\phi)(! \psi)$, this sort of update will typically introduce a CO of the form $O(\psi / \phi)$. Concretely: given an utterance of (1) at $c$, the associated CO (if the temperature drops, you must shut the window) will tend to be in force at the updated context, regardless of the truth of the temperature drops at either $c$ or $c$ updated with the CI.

A preliminary diagnosis of the problem: for CIs, we require an update on TDLs that is performed regardless of the antecedent's truth then. The failure seems to stem from deferring update to the ordering source until the antecedent of the imperative is true. An immediate thought, then, is to treat conditional imperative force as a kind of unconditional imperative force: 
Definition 3. $c[$ (if $\phi)(! \psi)]=c^{\prime}$ is just like c, except $\llbracket \phi \supset \psi \rrbracket^{c}$ is on $T_{c^{\prime}}\left(a_{c}\right)$

Call this the Wide-Scoping Proposal for CIs (WSPCI), so named because, according to the WSPCI, $[($ if $\phi)(! \psi)]=[!(\phi \supset \psi)][5$ The WSPCI runs into empirical problems. Consider the following case (from Kolodny and MacFarlane [7]): ten miners are trapped in a single shaft-A or B, although we do not know whichand threatened by rising waters. We can block one shaft or neither, but not both. If we block the shaft they are in, all are saved. If we guess wrong, all die. But, if we do nothing, water will distribute between the shafts and exactly one will die. Now consider the following set of imperatives.

(3) If they're in A, block $\mathrm{A} ! \approx\left(\right.$ if $\left.i n \_A\right)($ !block_A $)$

(4) If they're in B, block B! $\approx\left(\right.$ if $\left.i n_{-} B\right)($ !block_B $)$

(5) Don't block either shaft! $\approx ! \neg($ block_A $A$ block_B $)$

The imperatives in (3) seem like sound advice. But if the WSPCI is right, they add the following to the addressee's TDL: $\llbracket i n_{-} A \supset$ block $\_A \rrbracket^{c}$, $\llbracket i n_{-} B \supset$ block_B $B \rrbracket^{c}$, and $\llbracket \neg\left(b_{0} l_{c} \__{-} A \vee b l o c k_{-} B\right) \rrbracket^{c}$. The only way to satisfy all of these demands is to make sure the miners are in neither A nor B. But this is presupposed impossible at $c$. This does not square with intuitions: a speaker issuing these imperatives at $c$ is not demanding the impossible 6

\section{$3 \quad$ Restricting Force}

To summarize, the dynamic account, as it stands, does well with UIs, but fails to predict the relevant phenomena for CIs. Why? There are only two sorts of update to perform on a TDL: deferred and non-deferred (immediate) addition. Deferred addition, we saw, cannot account for the conventional discourse effect of CIs. Immediate addition implies that there is some proposition that a CI adds to the addressee's TDL - that conditional commanding is a species of unconditional commanding. There are no obvious candidates for the identity of this proposition.

Each tack presupposes that imperative force involves a speaker demanding that some proposition be true (with the deferred update proposal making this demand contingent on some further condition). I see no way of predicting the relevant phenomena for CIs while preserving this assumption. So we will jettison it. The guiding idea here will be a familiar one: unconditional commanding is a species of conditional commanding. The former corresponds to a kind of unrestricted imperative force, the latter to a kind of restricted imperative force.

\footnotetext{
${ }^{5}$ Note: allowing ! to take widest scope in CIs lets us handle CIs with quantificational adverbials in consequent position. Consider the CI if your boss comes in, never stare at him. Schwager [16] assigns this sentence a wide-scope LF: the antecedent restricts the domain of the quantificational adverbial, and the necessity modal takes scope over the adverbial. Schwager takes this to be evidence for the modal analysis, but simply allowing ! to take widest scope (thus allowing the conditional antecedent to subsequently restrict the adverbial) lets us mimic her analysis.

${ }^{6}$ People do quibble with this judgment. Kolodny and MacFarlane [7] argue that they are mistaken. Space prevents me from rehearsing the arguments here.
} 


\subsection{First Pass}

How to formalize this idea? The first thought is to type TDLs as Kratzer-ian conversational backgrounds: functions from worlds to a set of propositions. Doing this allows us to think of TDLs as something like a set of contingency plans: they furnish different practical 'recommendations' depending on the situation the agent finds herself in. Formally, we index TDLs to both agents and worlds, and treat (if $\phi)(! \psi)$ as adding $\llbracket \psi \rrbracket^{c}$ to $a_{c}$ 's TDL at the $\phi$-worlds (or some contextually selected subset thereof; cf. Mastop [12]: 103).

Definition 4. $c[($ if $\phi)(! \psi)]=c^{\prime}$ is like c, except $\forall w \in \llbracket \phi \rrbracket^{c}: \llbracket \psi \rrbracket^{c} \in T_{c^{\prime}}\left(a_{c}\right)(w)$

This is a natural and elegant extension of Kratzer's restrictor analysis of conditional antecedents. Rather than restricting the domain of a generalized quantifier, however, CI antecedents function to restrict the scope of dynamic update. Update with UIs is thus understood in terms of update with CIs, rather than vice versa. UIs issue a demand on the addressee that holds in all possible contingencies, while genuine CIs issue a demand on the addressee that holds in some non-trivial restriction of the set of possible contingencies.

Elegant though it is, this proposal does no better at predicting the desired relationship between CIs and COs. We do get unconditional obligations (UOS) of the form $O(\psi / \top)$ when evaluating these formulas at $\phi$-worlds. But we get nothing at $\neg \phi$-worlds. For the CI updates the addressee's TDL only at the $\phi$-worlds, and does nothing otherwise. Thus, we have only a metalinguistic analogue of the desired prediction: given that (if $\phi)(! \psi)$ is issued at $c$, if $\phi$ is true at $w$, then typically $\psi$ is required at $w$ (i.e., typically, $\llbracket O(\psi / \top) \rrbracket^{c^{\prime}, w}=1$, where $c^{\prime}$ is $c$ updated with (if $\phi)(\psi)$ ). This isn't good enough: we'd like to predict the objectlanguage $\mathrm{CO}$ if the temperature drops, you must shut the window true at the updated context, regardless of whether the temperature drops is true then 7

\subsection{Second Pass}

Something is very intuitive about the contingency plan understanding of the TDL. The problem is that, on the standard Kratzer semantics for modals, the world of evaluation fixes the ordering source at a context: contingencies cease to be relevant (in the sense that they are ignored by the semantics) once the world of evaluation is fixed. Avoiding this, then, demands that TDLs be indexed to some semantic parameter other than the world of evaluation.

Our analysis indexes TDLs to bodies of information (modal bases, whether construed as sets of worlds or propositions), rather than worlds. On this picture, the contingencies relevant to planning are informational, rather than 'factual,' in character: the TDL furnishes different practical 'recommendations' for an agent depending on the information available to her at the context. Formally, we treat

\footnotetext{
${ }^{7}$ We could predict the right relationship between CIs and cos by rewriting the Kratzer semantics as a strict conditional semantics, so that $\llbracket O(\phi / \psi) \rrbracket^{c, w}=1$ iff $\forall v \in \llbracket \phi \rrbracket^{c}$ : $\llbracket O(\psi / \top) \rrbracket^{c, v}=1$. But this seems like an ad hoc revision of the semantics.
} 
(if $\phi)(! \psi)$ as adding $\llbracket \psi \rrbracket^{c}$ to $a_{c}$ 's TDL at every body of information $\Phi \supseteq f_{c}(w) \cup$ $\left\{\llbracket \phi \rrbracket^{c}\right\}$, for each $w$ (i.e., every $\phi$-containing expansion of the information at $c$ ).

Definition 5. $c[($ if $\phi)(! \psi)]=c^{\prime}$ is like c, except:

$\forall w: \forall \Phi \supseteq f_{c}(w) \cup\left\{\llbracket \phi \rrbracket^{c}\right\}: \llbracket \psi \rrbracket^{c} \in T_{c^{\prime}}\left(a_{c}\right)(\Phi)$

As before, a UI ! $\phi$ is a vacuously restricted CI: $[! \phi]:=[($ if $\top)(! \phi)]$; unconditional commanding is still a species of conditional commanding. The difference is that UIs add their consequents to every expansion of the information sans phrase.

This is the analysis of imperative force which I will be endorsing in this paper. It is a restrictor analysis of CI antecedents: CI antecedents restrict the set of contingencies to which a command pertains, thereby modulating the force of the associated speech-act. It represents an abandonment of the 'propositional attitude' model of speech acts described in this essay's introduction.

\subsection{Information-Sensitive Ordering Semantics}

Allowing the ordering source at a context $c$ to be determined by the information at $c$, rather than the world of evaluation, does not, by itself, secure the desired relationship between CIs and COs. Getting this right requires modifying the semantics to make use of the information-sensitive ordering source 8 The relevant change is having conditional antecedents function as both domain restrictors and ordering source shifters, so that the if-clause supplies the relevant contingency:

Definition 6. $\llbracket O(\psi / \phi) \rrbracket^{c, w}=1 \Leftrightarrow \min \left(f_{c}(w) \cup\left\{\llbracket \phi \rrbracket^{c}\right\}, \preceq_{T_{c}(a)\left(f_{c}(w) \cup \llbracket \phi \rrbracket^{c}\right)}\right) \subseteq \llbracket \psi \rrbracket^{c}$

Informally, the formula $O(\psi / \phi)$ says the best-on-the supposition-that- $\phi \phi$-worlds are $\psi$-worlds. This secures the right result in the if the temperature drops... case. The relevant CI adds the proposition that the addressee shuts the window to her TDL at every body of information $\Phi$ such that $\Phi$ entails that the temperature is dropping. The information-sensitive semantics (ISS) evaluates the relevant CO by looking at the addressee's TDL with respect to such a body of information.

This is a major revision of the Kratzer 8] semantics, which allows contingency in ordering sources only via variation in the world coordinate, not via variation in the domain of quantification. So there is reason to worry that it is ad hoc. It can, in fact, be independently motivated. Consider, once again, Kolodny and MacFarlane's [7] miner case, and the obligation-descriptions in (6] 8).

(6) If they're in $\mathrm{A}$, we gotta block $\mathrm{A} \approx O($ block_A/in_A)

(7) If they're in $\mathrm{B}$, we gotta block $\mathrm{B} \approx O\left(b l o c k \_B / i n_{-} B\right)$

(8) We may leave both shafts open $\approx \neg O(($ block_A $A \vee$ block_B $) / \top)$

Given the case, informants reliably hear each of these obligation-descriptions as true (so, a fortiori, consistent). But, using the information-insensitive Kratzer semantics, whenever the modal base entails (i.e., it is known) that the miners are either all in $\mathrm{A}$ or all in $\mathrm{B}$, these sentences are provably inconsistent.

\footnotetext{
8 The issues here are discussed in more detail in my [3]
} 
Proof. Suppose $\llbracket(6) \rrbracket^{c, w}=\llbracket(17) \rrbracket^{c, w}=\llbracket(8) \rrbracket^{c, w}=1$ and $\cap f_{c}(w) \subseteq \llbracket i n_{-} A \vee i n_{-} B \rrbracket^{c}$.

1. Let $g_{c}$ be an ordering source. Choose any $v \in \min \left(f_{c}(w), \preceq g_{c}(w)\right)$.

2. Since $\bigcap f_{c}(w) \subseteq \llbracket i n_{-} A \vee i n_{-} B \rrbracket^{c}, v \in \min \left(f_{c}(w) \cup\left\{\llbracket i n_{-} A \rrbracket^{c}\right\}, \preceq g_{c}(w)\right)$ or $v \in \min \left(f_{c}(w) \cup\left\{\llbracket i n_{-} B \rrbracket^{c}\right\}, g_{g_{c}(w)}\right) 9$

3. By Kratzer's semantics (Def. 1), since 【(6) $\rrbracket^{c, w}=\llbracket(7) \rrbracket^{c, w}=1, \min \left(f_{c}(w) \cup\right.$ $\left\{\llbracket i n \_A \rrbracket^{c}\right\}, \preceq g_{c}(w) \subseteq \llbracket b l o c k \_A \rrbracket^{c}$ and $\min \left(f_{c}(w) \cup\left\{\llbracket i n_{-} B \rrbracket^{c}\right\}, \preceq g_{c}(w)\right) \subseteq \llbracket b l o c k \_B \rrbracket^{c}$.

4. So $v \in \llbracket b l o c k \_A \rrbracket^{c} \cup \llbracket b l o c k \_B \rrbracket^{c}$.

5. So $\min \left(f_{c}(w), \preceq_{c}(w)\right) \subseteq \llbracket b l o c k \_A \rrbracket^{c} \cup \llbracket b l o c k \_B \rrbracket^{c}$.

6. So, by Def. 1, $\llbracket O\left(\left(b l o c k_{-} A \vee b l o c k_{-} B\right) / \top\right) \rrbracket^{c, w}=1$. Contradiction.

Space prevents me from discussing in detail the substance of the the proof or our proposal for COs. Briefly, the ISs blocks the proof by varying the ordering sources that are relevant for evaluating the CO-descriptions in (6, [6): (6) uses an ordering source indexed to a body of information that entails that the miners are in shaft A, (7) uses an ordering source indexed to a body of information that entails that the miners are in shaft B, while (8) uses an ordering source indexed to a body of information that does not settle the miners' location 10 The upshot: the ISS seems to be independently motivated, not ad hoc.

\section{Sequencing}

We may think of imperative force as a complex update on TDLs, constructed out of a set of basic updates on TDL components, together with a regular operation. In this case, the operation is ; (SEQUENCING). Sequencing is function composition: if $\alpha$ and $\beta$ are context-change potentials, then $\alpha ; \beta=\lambda c . c \alpha \beta$. A TDL is a set of contingency plans: a set of information-plan pairs. The basic updates are additions to contingency plans. Complex or composite update is understood as a series of additions to an addressee's contingency plans (which being a function of how the speaker modulates the force of her command). The update associated with a $\mathrm{CI}($ if $\phi)(! \psi)$ at $c$ is a sequencing of the following basic update program:

$$
\lambda\langle\Phi, \Psi\rangle .\left\{\begin{array}{l}
\left\langle\Phi, \Psi \cup\left\{\llbracket \psi \rrbracket^{c}\right\}\right\rangle \text { if } f_{c}(w) \cup \llbracket \phi \rrbracket^{c} \subseteq \Phi \\
\langle\Phi, \Psi\rangle \text { otherwise }
\end{array}\right.
$$

\footnotetext{
${ }^{9}$ This step relies on a kind of montonicity property of the Kratzer semantics: if $u \in$ $\min (\Phi, \preceq)$, then for any $\Psi$ such that $\bigcap \Psi \subseteq \bigcap \Phi$ and $u \in \bigcap \Psi, u \in \min (\Psi, \preceq)$.

10 There is, I argue in my [3], a decision-theoretic motivation for allowing considerations of value (i.e., the elements of a deontic ordering source) to make a difference in determining what an agent should do only when they meet informational constraints: they must be actionable (roughly, knowably realizable) with respect to the relevant information (for relevant precedents, see Weirich [18, particularly his remarks on conditional utility, and Hawthorne and Stanley [6]). This both (i) motivates a semantics for deontic $O$ on which its ordering source is information-sensitive, (ii) accounts for the variation in the ordering sources that blocks the proof.
} 
There is, then, a sense in which utterances of CIs conventionally involve the performance of a composite speech-act: a 'conjunction' of instructions about updating individual contingency plans 11 Standard treatments of force do not provide for complex updates built with regular operations: force is computed by applying a force-operator to a content, and doesn't embed.

We also see speech-act sequencing, of a rather different sort, in the various ways a speaker may direct imperative force. So far we have (implicitly) construed imperatives as taking direction arguments: a context in which an imperative utterance occurs will tend to select someone at whom imperative force is targeted, i.e., an addressee. This orientation is, we see, sufficiently flexible to distinguish singular-addressee imperatives like (9) from group-addressee imperatives like (10). But it founders with plural-addressee imperatives like (11) and (12).

(9) Have the orchestra play Beethoven's $5^{\text {th }} \approx$ ! (make-play $\left.(\mathbf{5 t h})(\mathbf{o r c h})\left(a_{c}\right)\right)$

(10) Play Beethoven's $5^{\text {th }}$ (together $) \approx !\left(\operatorname{play}(\mathbf{5 t h})\left(a_{c}\right)\right)\left(a_{c}=\right.$ the orchestra $)$

(11) Everyone play her part $\approx$ ???

(12) (Conductor addressing orchestra members:) Play your part $\approx$ ???

In (11) and (12) no single individual or group of individuals is targeted by the imperative. Rather, each individual in a set of addressees is targeted, separately. Their force is, for each addressee $a$, to instruct that $a$ play $a$ 's part.

Plural addressee imperatives seem to involve some sort of quantification outscoping the imperative operator. So they demand a representation something like the following: $\forall x$ ! [play (the-part-of $-x)(x)] 12$ In the absence of a vocative (as with, e.g., 12), the default approach will be to bind free variables by $\forall$-closure. The intended interpretation of such formulas has them denoting sequences of updates: the result of sequencing the following set of updates, for all $a \in \mathcal{A}_{c}$, where $\mathcal{A}_{c}$ is the set of addressees determined by $c$ :

$$
\left\{\beta \mid \exists a \in \mathcal{A}_{c}: \beta=[!(\text { play }(\text { the- } \operatorname{part}-\text { of }-x)(x))]^{[x / a]}\right\}
$$

In the general case, formulas of the form $\forall x$ : (if $\phi)(! \psi)$ are interpreted as in Def. 7. (We assume, implicitly, that quantification into an imperative operator must always bind a variable in the direction-argument-position.)

Definition 7. $c[\forall x:($ if $\phi)(! \psi)]=c^{\prime}$ is like $c$, except: $\forall a \in \mathcal{A}_{c}: \forall w: \forall \Phi \supseteq f_{c}(w) \cup\left\{\llbracket \phi \rrbracket^{c}\right\}: \llbracket \psi \rrbracket^{c_{[x / a]}} \in T_{c^{\prime}}(a)(\Phi)$

Formulas of the form $\forall x$ : (if $\phi)(! \psi)$ can thus be viewed as expressing sequences of speech-acts along two dimensions. We have a 'conjunction' of instructions for the addressees, and each such instruction for a given addressee $a$ is comprised of a 'conjunction' of instructions about updating $a$ 's contingency plans.

${ }^{11} \mathrm{Cf}$. the dynamic treatment of conjunction as function-composition: $\sigma[\phi \wedge \psi]=\sigma[\phi][\psi]$. On this view, $[\phi \wedge \psi]$ is a composite speech-act - the result of sequencing $[\phi]$ and $[\psi]$.

${ }^{12}$ Cf. Krifka [9, 10, who argues for representing pair-list readings of questions with universal quantification into questions. 
The general orientation of this approach raises further questions. I can only gesture at their answers here. For instance: is embedding of speech-act-operators under an $\exists$-like operation (or of update potentials under a $\vee$-like operation) permitted (cf. Krifka [10, who suggests that it may be)? My tentative answer is: probably not. The purported evidence for the expressibility of such speechacts in natural language is weak (see my [2]). There is, moreover, arguably no reasonable thing for such an operation to mean. Suppose basic update potentials are functions defined for contexts. Interpreting $V$ in terms of $U$ will tend to yield update potentials that are not functions from input contexts to output contexts, but rather relations between input contexts and several possible output contexts. Complex update potentials formed with such operations will tend, in other words, to be indeterministic programs. Indeterminism in update potentials is prima facie objectionable: basic conversational platitudes plausibly require that a cooperative speaker know how her utterance will update the context 13

\section{References}

[1] Asher, N., Lascarides, A.: Indirect Speech Acts. Synthese 128, 183-228 (2001)

[2] Charlow, N.: Directives. Ms (2009a)

[3] Charlow, N.: What we know and what to do. Ms (2009b)

[4] Groenendijk, J., Stokhof, M.: Studies on the semantics of questions and the pragmatics of answers. Ph.D. Diss., ILLC (1984)

[5] Han, C.: The structure and interpretation of imperatives: Mood and force in universal grammar. Ph.D. Diss. University of Pennsylvania (1998), http://www.sfu.ca/ chunghye/papers/dissertation.pdf

[6] Hawthorne, J., Stanley, J.: Knowledge and action. The Journal of Philosophy 105, 571-590 (2008)

[7] Kolodny, N., MacFarlane, J.: Ifs and oughts. Ms (2009), http://johnmacfarlane.net/ifs-and-oughts.pdf

[8] Kratzer, A.: The notional category of modality. In: Eikmeyer, H., Rieser, H. (eds.) Words, Worlds, and Contexts, pp. 38-74. De Gruyter, Berlin (1981)

[9] Krifka, M.: Quantifying into question acts. Natural Language Semantics 9, 1-40 (2001)

[10] Krifka, M.: Semantics below and above speech acts. Talk delivered at Stanford (2004), http://amor.rz.hu-berlin.de/ h2816i3x/Talks/ StanfordLecture2004.pdf

[11] Lewis, D.: General semantics. Synthese 22, 18-67 (1970)

[12] Mastop, R.: What can you do? Ph.D. Diss., ILLC (2005)

[13] Portner, P.: The semantics of imperatives within a theory of clause types. In: Watanabe, K., Young, R. (eds.) Proceedings of SALT 14. CLC Publications (2004), http://semanticsarchive.net/Archive/mJlZGQ4N/

[14] Portner, P.: Imperatives and modals. Natural Language Semantics 15, 351-383 (2007)

$\overline{13}$ There are possible interpretations for $\vee$ that preserve determinism. For instance, 'disjoined' speech-acts might map a context into a set of alternative contexts (cf. Mastop [12]). But this gets formally unwieldy very quickly (see esp. Krifka [10]). 
[15] Potts, C.: Keeping world and will apart: A discourse-based semantics for imperatives. NYU Syntax/Semantics Lecture Series (2003), http://people.umass.edu/potts/talks/potts-nyu-handout.pdf

[16] Schwager, M.: Conditionalized imperatives. In: Gibson, M., Howell, J. (eds.) Proceedings of SALT 16. CLC Publications (2006), http://user.uni-frankfurt.de/ scheiner/papers/schwagerFEB07.pdf

[17] Stenius, E.: Mood and language game. Synthese 17, 254-274 (1967) doi:10.1007/BF00485030

[18] Weirich, P.: Decision when desires are uncertain. In: Bradie, M., Sayre, K. (eds.) Reason and Decision, pp. 69-75. Bowling Green State UP (1982) 